ARTICLE

\title{
Methylotrophic methanogenic Thermoplasmata implicated in reduced methane emissions from bovine rumen
}

Morten Poulsen¹, Clarissa Schwab², Bent Borg Jensen ${ }^{1}$, Ricarda M. Engberg${ }^{1}$, Anja Spang ${ }^{2}$, Nuria Canibe ${ }^{1}$ Ole Højberg ${ }^{1}$, Gabriel Milinovich², Lena Fragner ${ }^{3}$, Christa Schleper², Wolfram Weckwerth ${ }^{3}$, Peter Lund ${ }^{1}$, Andreas Schramm ${ }^{4} \&$ Tim Urich ${ }^{2}$

Rumen methanogens are major sources of anthropogenic methane emissions, and these archaea are targets in strategies aimed at reducing methane emissions. Here we show that the poorly characterised Thermoplasmata archaea in bovine rumen are methylotrophic methanogens and that they are reduced upon dietary supplementation with rapeseed oil in lactating cows. In a metatranscriptomic survey, Thermoplasmata 16S rRNA and methylcoenzyme $M$ reductase $(\mathrm{mcr}$ ) transcripts decreased concomitantly with mRNAs of enzymes involved in methanogenesis from methylamines that were among the most abundant archaeal transcripts, indicating that these Thermoplasmata degrade methylamines. Their methylotrophic methanogenic lifestyle was corroborated by in vitro incubations, showing enhanced growth of these organisms upon methylamine supplementation paralleled by elevated methane production. The Thermoplasmata have a high potential as target in future strategies to mitigate methane emissions from ruminant livestock. Our findings and the findings of others also indicate a wider distribution of methanogens than previously anticipated.

\footnotetext{
${ }^{1}$ Department of Animal Science, Aarhus University, Blichers allé 20, 8830 Tjele, Denmark. ${ }^{2}$ Department of Genetics in Ecology, University of Vienna, Althanstrasse 14, Vienna 1090, Austria. ${ }^{3}$ Department of Molecular Systems Biology, University of Vienna, Althanstrasse 14, Vienna 1090, Austria.

${ }^{4}$ Department of Bioscience, Aarhus University, Ny Munkegade 116, 8000 Aarhus, Denmark. Correspondence and requests for materials should be addressed to M.P. (email: Morten.Poulsen@agrsci.dk) or to T.U. (email: Tim.Urich@univie.ac.at).
} 
R uminant livestock is a major global source of methane $\left(\mathrm{CH}_{4}\right)^{1,2}$. The digestive fermentation activity of ruminants accounts for $\sim 37 \%$ of anthropogenic $\mathrm{CH}_{4}$ emissions ${ }^{2}$. $\mathrm{CH}_{4}$ is the end product of a complex anaerobic degradation pathway of plant biomass by the ruminal microbiota, the terminal group being methanogenic archaea. $\mathrm{H}_{2}$ is the major energy source of rumen methanogens known to date ${ }^{3}$, and these archaea are targets in strategies aimed at reducing $\mathrm{CH}_{4}$ emissions. With global demands for milk and meat expected to double by 2050, $\mathrm{CH}_{4}$ emissions are predicted to increase accordingly ${ }^{4}$. Ruminal $\mathrm{CH}_{4}$ production also represents an energy loss to the animal ${ }^{5}$; hence, reducing methanogenesis is of both environmental and economic interest.

Until recently, rumen methanogens have been found to belong to a few genera of the Methanobacteriales and Methanomicrobiales orders within the phylum Euryarchaeota ${ }^{3}$. These orders have all been characterised as hydrogenotrophic, utilizing $\mathrm{H}_{2} / \mathrm{CO}_{2}$ or $\mathrm{H}_{2} /$ methanol produced by various fermentative bacteria in the process of anaerobic degradation of plant biomass. A novel group ${ }^{6}$ distantly related to the Thermoplasmatales (recently named 'rumen cluster C' $(\mathrm{RCC})^{3}$; previously described as rice cluster $\mathrm{C}$ Thermoplasmata ${ }^{7}$ ) was found to be highly abundant in ruminants based on $16 \mathrm{~S}$ ribosomal RNA (rRNA) genes ${ }^{3}$. No representatives of the RCC Thermoplasmata have been isolated, although their methanogenic lifestyle was proposed because of the parallel presence of distinct methyl-coenzyme $M$ reductase genes $(m c r)^{3,8,9}$. However, the recently described Methanomassiliicoccus luminyensis isolated from human feces from the related rice cluster III clade provided evidence for methanogenic Thermoplasmata ${ }^{10}$.

Approaches to reduce $\mathrm{CH}_{4}$ emission from livestock, such as dietary supplementation of unsaturated fatty acids ${ }^{11}$, typically focus on reducing $\mathrm{H}_{2}$ availability for rumen methanogens. However, because of the complexity of the rumen microbiota, results are not fully predictable and the effects of the manipulations are poorly understood ${ }^{12}$.

Here, for the first time, a metatranscriptomic approach was used to investigate the in vivo effect of rapeseed oil (RSO) supplementation on the rumen microbiota of lactating Holstein cows, with particular focus on methanogenic archaea. We show that the poorly characterised Thermoplasmata in bovine rumen are a novel group of methylotrophic methanogens and that they are reduced upon $\mathrm{CH}_{4}$ mitigation in lactating cows.

\section{Results}

Effect of RSO supplementation on $\mathrm{CH}_{4}$ emission. RSO supplementation significantly reduced $\mathrm{CH}_{4}$ emission, as shown by a $6.2 \%$ lower $\mathrm{CH}_{4}$-to- $\mathrm{CO}_{2}$ emission ratio $(P<0.05$; Supplementary Table S1). In a previous experiment with identical diets, $\mathrm{CH}_{4}$-to$\mathrm{CO}_{2}$ ratio was reduced by $8.5 \%$ upon $\mathrm{RSO}$ supplementation, corresponding to an $18 \%$ reduction in emitted $\mathrm{CH}_{4}$ per energy corrected milk yield ${ }^{13}$ (Supplementary Table S1).

Rumen metatranscriptomics. A metatranscriptomics approach with random-hexamer primed reverse transcription was applied on rumen fluid to simultaneously assess the impact of RSO on the abundance of rumen microorganisms and their gene expression ${ }^{14}$ (see Methods). Illumina deep-sequencing with overlapping read paired-end technology ${ }^{15}$ resulted in $\sim 10-12$ million high-quality reads of $\sim 160$-bp length per sample. Although the majority of sequences represented rRNA ('ribo-tags'), between 380,000 and 485,000 sequences were putative messenger RNAs (mRNAs) (Supplementary Table S2).

A three-domain profile of the rumen microbiota revealed that Bacteria and Eukaryotes each contributed approximately $50 \%$ of ribo-tags, whereas Archaea were present at approximately 1\% (Supplementary Fig. S1; see Supplementary Figs. S2 and S3 for a complete overview of bacterial and eukaryotic communities, respectively). The Archaea was constituted by Methanobacteriales (genera Methanobrevibacter and Methanosphaera) and the RCC Thermoplasmata. Surprisingly, only the RCC decreased significantly $(P<0.05)$ with RSO supplementation (Fig. 1), indicating that they were associated with the reduction in $\mathrm{CH}_{4}$, whereas the well-studied Methanobacteriales were not. Likewise, the mRNA levels of the key-enzyme methyl-coenzyme M reductase (Mcr) were reduced upon RSO amendment (Fig. 1). Remarkably, transcripts of all three subunits $\alpha, \beta$ and $\gamma$ of $\mathrm{Mcr}$
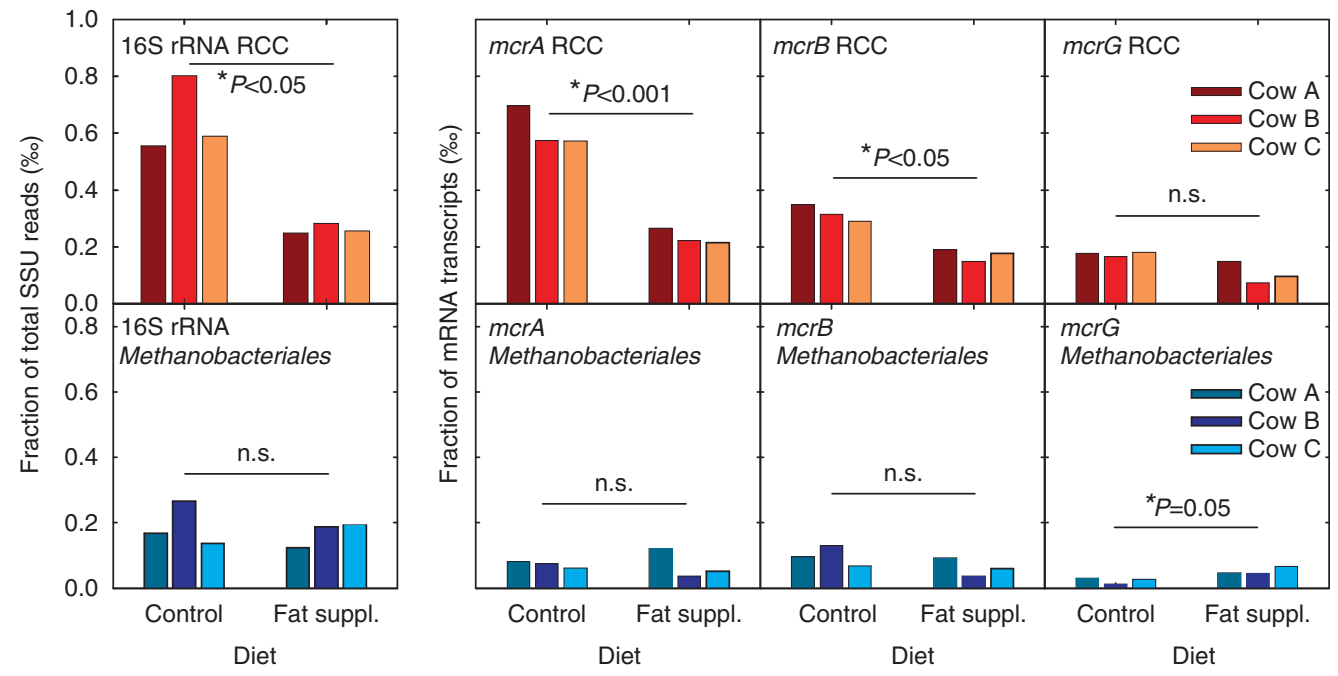

Figure 1 | Effect of RSO on methanogens in vivo. Relative abundances of transcripts from RCC (upper row) and from Methanobacteriales (bottom row) from individual cows on control and rapeseed oil (RSO)-supplemented diets, respectively. Relative fraction of archaeal SSU ribo-tags affiliated to RCC and Methanobacteriales is shown as \% of total SSU reads, and fraction of $m c r A$, mcrB and mcrG transcripts detected for RCC and other methanogens (that is, Methanobrevibacter ruminantium and Methanosphaera stadtmanae), shown as \%o of all mRNA transcripts with a significant homologue in Genbank nr (e-value $<1 e-5)$ for each sample. $P$-values $\leq 0.05$ are considered representing significant effects of fat supplementation $\left({ }^{\star}\right.$ marked) using paired $t$-test. The $\mathrm{mcrG}$ of RCC was trend-wise affected by fat supplementation $(P<0.10)$. n.s., not significant; suppl, supplement. 
were affected (Fig. 1). The decrease of $m c r A$ transcripts was corroborated by quantitative PCR (qPCR) (Supplementary Table 3).

Mcr transcript assembly using the CAP3 program resulted in full-length sequences of the three mor subunits $\alpha, \beta$ and $\gamma$, among shorter contigs. Although several of the short contigs were highly similar to $\mathrm{mcr}$ subunits of Methanobrevibacter and Methanosphaera, the long contigs were only distantly related to mor of Methanobrevibacter and Methanosphaera or to other cultured methanogens ( $<70 \%$ DNA similarity) (Fig. 2a). However, $m c r A$ was $>95 \%$ similar to partial $m c r A$ genes associated with the RCC archaea (for example Evans et al. ${ }^{9}$ and Acc. nos. JN229252/JN229233). Based on this, all three subunits were assigned to RCC. We validated this approach through assembly of the archaeal transcripts of elongation factor $1 \alpha$ $(\mathrm{EF}-1 \alpha)$ and $16 \mathrm{~S}$ rRNA. Phylogenetic reconstruction of a translated amino-acid sequence of the obtained full-length EF$1 \alpha$ revealed an association with EF- $1 \alpha$ of the Thermoplasmata branch of Euryarchaota (Fig. 2b), which is similar to the placement of the 16S rRNA of RCC (Fig. 2c). Assignment of all single $\mathrm{mor}$ transcripts to the full-length $\mathrm{mcr}$ genes of the RCC clade and the Methanobacteriales revealed a significant decrease of $m c r A$ and $m c r B$ transcripts of RCC upon RSO amendment $(P<0.05$; Fig. 1), and not of the Methanobacteriales. Taken together with the $16 \mathrm{~S}$ rRNA data, this gives evidence that the RCCs were responsible for the decreased $\mathrm{CH}_{4}$ emissions.
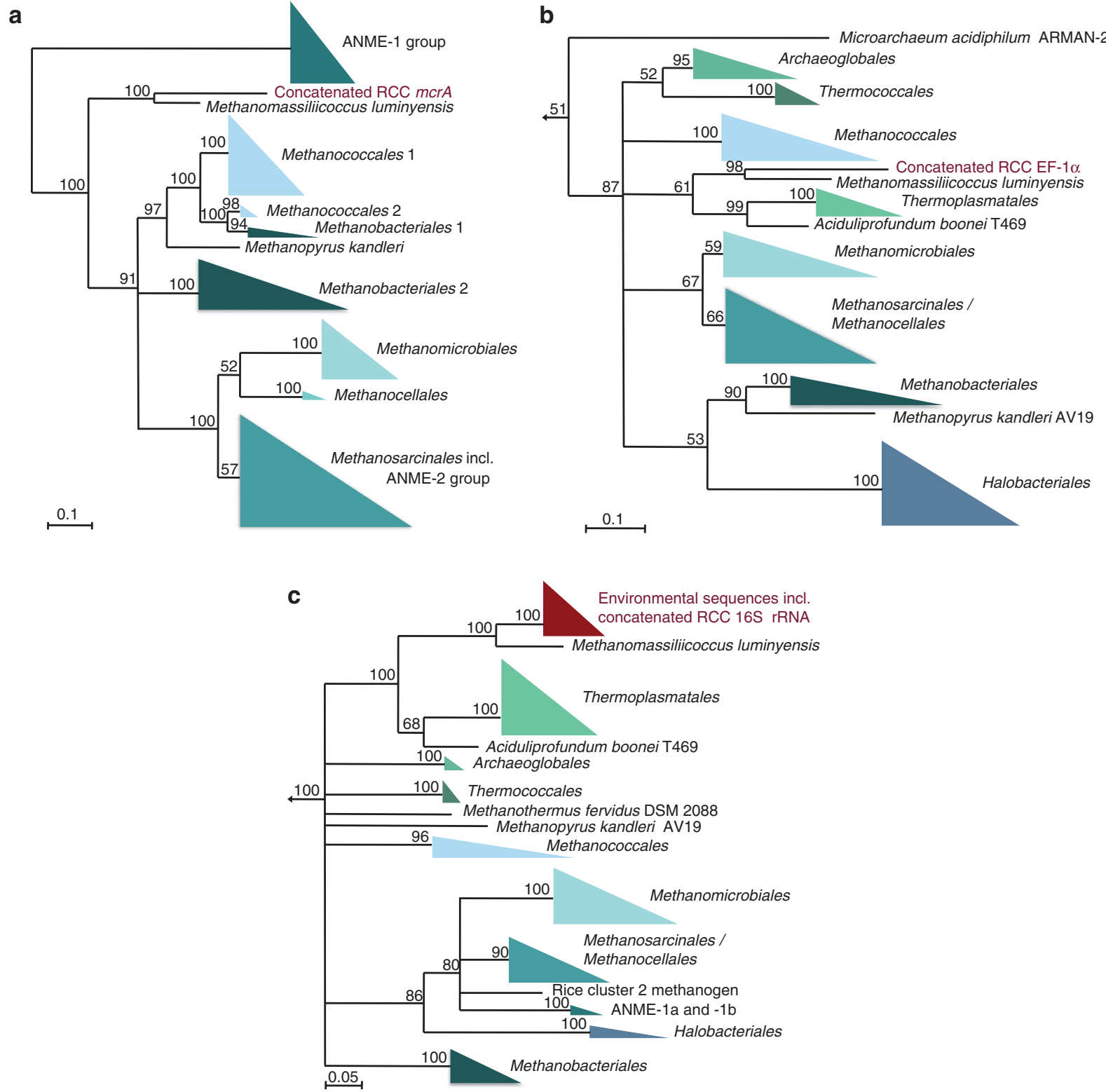

Figure 2 | Phylogenetic analysis of RCC Thermoplamata. Maximum likelihood phylogenetic analyses of concatenated $m c r A$, EF-1 $\alpha$ and 165 rRNA sequences of RCC. (a) McrA phylogeny based on an amino-acid alignment (569 positions after removal of ambigiously aligned sites and 100 bootstraps). The scale bar refers to $10 \%$ estimated sequence divergence. (b) EF- $1 \alpha$ phylogeny based amino-acid alignment (368 positions) (removal of ambiguously aligned sites, 50\% indel filter and 100 bootstraps). The tree was rooted with corresponding sequences from Cren-, Kor- and Thaumarchaeota. The scale bar refers to 10\% estimated sequence divergence. (c) 16S rRNA phylogeny based on nucleotid alignment (1212 positions) (removal of ambigiously aligned sites and 100 bootstraps). Thaumarchaeal $16 \mathrm{~S}$ rRNA sequences were used as outgroup. The scale bar refers to $5 \%$ sequence divergence. Nodes with bootstrap value $>50$ were kept, whereas those $<50$ were removed and are shown as multifurcations. 
Methylotrophic methanogenesis from methylamines (MAs). Although the RCC clade has been previously suggested as a novel order of methanogens $3,8,9$, information on the pathway of methanogenesis was not available. A global functional analysis of all mRNAs with MEGAN using the SEED functional categories $^{16}$ revealed the presence of transcripts for the methanogenic pathways with methylated compounds as substrates (Fig. 3a). These were similar in abundance to transcripts of the 'core methanogenesis', and likewise decreased significantly upon RSO treatment $(P<0.05$, Supplementary Fig. S4a). Closer inspection identified transcripts for utilization of tri-, di- and monomethylamine and methanol, pointing to these substances as important substrates for the rumen methanogens (Fig. 3b). Especially, the presence of transcripts for utilization of MAs is remarkable, because Methanosarcinaceae were until now the only methanogens known to grow on these substrates. Furthermore, all enzymes in the three MA pathways were only distantly related to those from Methanosarcinaceae ( $<66 \%$ amino-acid sequence identity, Supplementary Table S4). The first enzymes in these pathways are corrinoid-dependent MA methyltransferases $\left(\mathrm{Mtl}^{17}\right)$; these contain the unique, reactive amino-acid pyrrolysine (the $22^{\text {nd }}$ aa), encoded via an in-frame stop codon on the mRNA, as a conserved trait ${ }^{17}$. All putative $m t 1$ transcripts harboured this stop codon, making it highly likely that these indeed encode functional Mt1 enzymes. In summary, the metatranscriptomic data strongly suggest that RCCs are a novel order of methylotrophic methanogens, obtaining energy and carbon from MAs derived from the degradation of betaine and choline, and possibly also from methanol. Additional evidence for this methylotrophic methanogenic lifestyle comes from the recently isolated Methanomassiliicoccus luminyensis of the closely related rice cluster III, which was isolated with $\mathrm{H}_{2} /$ methanol as substrate. A screening of the M. luminyensis genome $^{18}$ with the RCC transcripts revealed the presence of all genes for methanogenesis from MA (Supplementary Table S4).
Also very recently, a methanogenic enrichment from the termite gut was dominated by Thermoplasmata utilizing $\mathrm{H}_{2} /$ methanol $^{19}$.

Methylotrophic methanogenesis from MA was verified in vitro by adding trimethylamine (TMA) to bovine rumen fluids. Incubation with $15 \mathrm{mM}$ TMA resulted in a $22 \%$ increase $(P<0.01)$ of $\mathrm{CH}_{4}$ in the headspace by $24 \mathrm{~h}$ (Fig. $\left.4 \mathrm{a}\right)$, whereas the addition of the methanogenesis inhibitor bromoethanesulfonic acid completely inhibited $\mathrm{CH}_{4}$ formation (Supplementary Fig. S5). This confirmed the ability of rumen methanogens to quickly utilize TMA. Abundance of RCC methanogens, as determined by qPCR of $16 \mathrm{~S}$ rRNA and TMA methyltransferase $(m t t B)$ genes, increased threefold in the presence of TMA. In contrast, growth of other methanogens was independent of TMA addition as indicated by comparable increase of 16S rRNA gene copies in both control and TMA supplemented samples, respectively (Fig. 4b,c and Supplementary Fig. S6).

Sources of MAs and rumen nitrogen cycling. Formation of TMA from glycine betaine and (phosphatidyl-) choline by rumen bacteria and of $\mathrm{CH}_{4}$ from MAs has been shown during the last decades $^{20-23}$. The identification of methylotrophic RCC finally identifies the responsible methanogens. Especially, glycine betaine is present in high amounts in feeds containing beet pulp, which was also included in the diet used in this study (see Supplementary Table S5 for details). This might also explain the comparably high abundance of RCC compared with other studies $^{3}$. As the degradation of MAs leads to the release of ammonium $\left(\mathrm{NH}_{4}^{+}\right)^{20,21}$, the utilization of MAs by RCC has implications for the entire rumen bacterial community. Intriguingly, the metatranscriptomic data also revealed a decrease of $30-60 \%$ of transcripts for $\mathrm{NH}_{4}^{+}$assimilation in the RSO-supplemented cows (Supplementary Fig. S4b), suggesting that RCC constitute an additional supplier of $\mathrm{NH}_{4}^{+}$in the rumen, besides $\mathrm{NH}_{4}^{+}$derived from protein degradation.

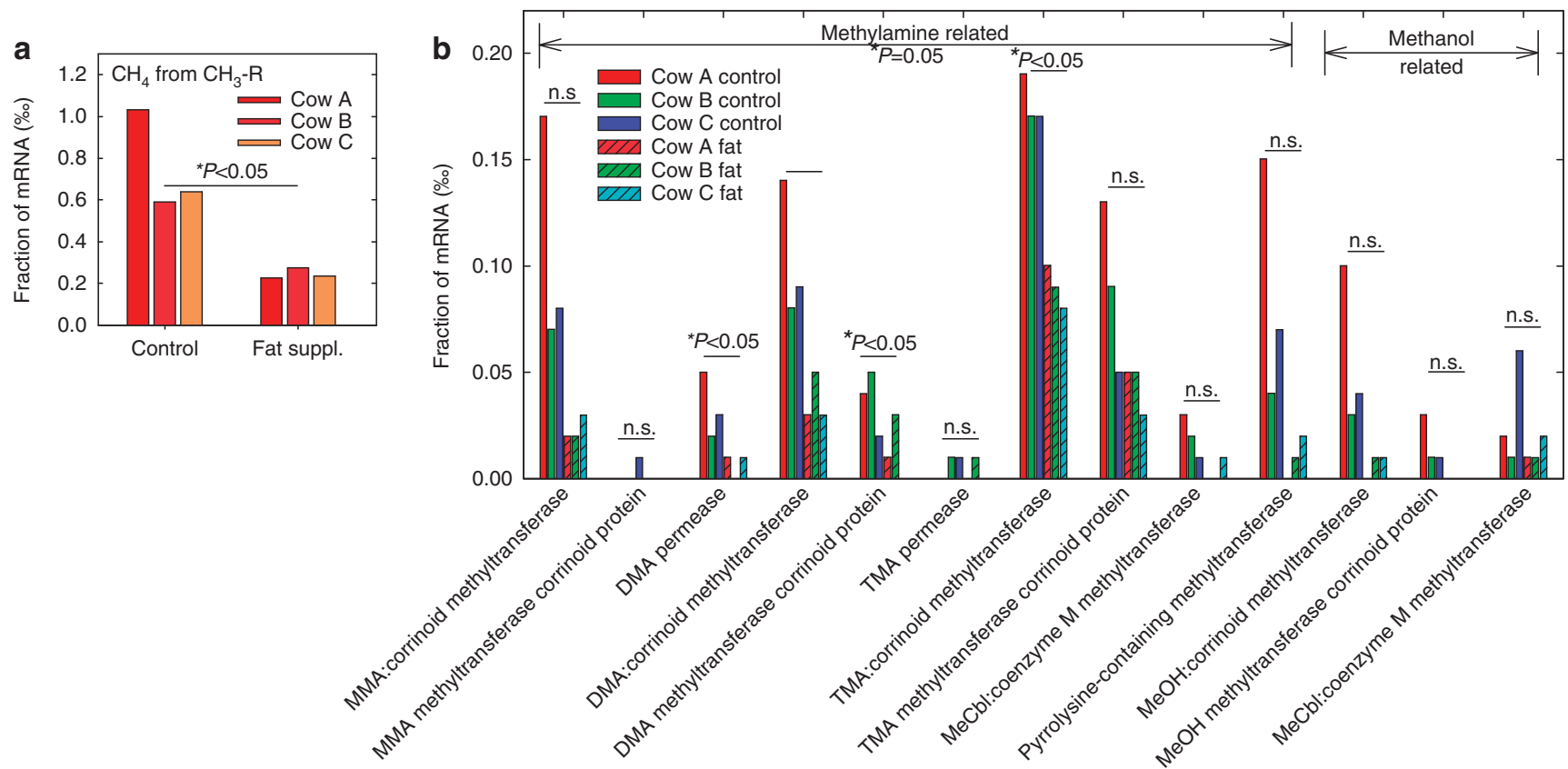

Figure 3 | Transcripts of methanogenesis from methylamines. Effect of RSO amendment on the expression of SEED functional gene categories of (a) methanogenesis from methylated compounds combined and (b) transcripts for utilizing MAs and methanol as substrates for $\mathrm{CH}_{4}$ production inferred from a global functional analysis of all mRNAs with MEGAN using the SEED functional categories. MMA, monomethylamine; DMA, dimethylamine; TMA, trimethylamine; $\mathrm{MeCbl}$, methylcobalamine; $\mathrm{MeOH}$, methanol. $P$-values $\leq 0.05$ are considered representing significant effects of fat supplementation ( ${ }^{\star}$ marked) using paired $t$-test. n.s., not significant; suppl, supplement. 


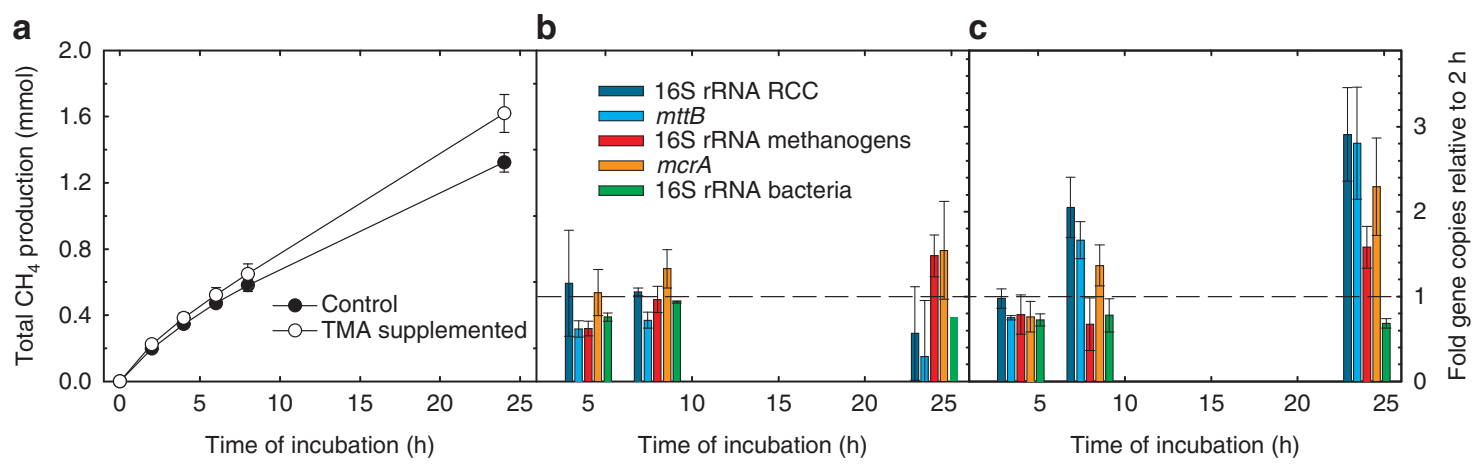

Figure 4 | Methanogenesis from TMA by $\mathbf{R C C}$ in vitro. $\mathrm{CH}_{4}$ production (a) without and with TMA (15 mM), and concurrent $x$-fold change in bacterial, methanogen and RCC $16 \mathrm{~S}$ rRNA genes, $m t t B$ and $m c r A$ in rumen fluid fermentations (b) without and (c) with TMA, as quantified by qPCR. X-fold change in gene copies was calculated relative to $2 \mathrm{~h}$. Total $\mathrm{CH}_{4}$ production differed significantly between treatments after $24 \mathrm{~h}$ (non-paired $t$-test; $P<0.01$ ). Error bars represent s.d. of quadruple incubations. TMA, trimethylamine; mttB, TMA methyltransferase; $m c r A$, methyl-coenzyme $M$ reductase subunit $\alpha$.

Inhibitory effect of RSO. In order to obtain an estimate about the concentration of MAs in the rumen fluids and to obtain insights into the inhibitory effect of RSO on RCC, we quantified TMA using direct infusion Orbitrap mass spectrometry (see Methods). The TMA concentrations were approximately $40 \mu \mathrm{M}$ in the controls and increased about fivefold to $220 \mu \mathrm{M}$ in RSO samples. These data show that (1) substantial amounts of TMA were present in the rumen fluids and (2) that TMA did not become limiting in the RSO samples. The latter indicates that the inhibitory effect of RSO on RCC is not via substrate limitation. Rather, RSO components (for example, glycosinolates) might have been directly or indirectly toxic for RCC. This conclusion is supported by the fact that no consistent change in the rumen bacterial community composition of replicate samples was observed with RSO amendment (Supplementary Fig. S2), as it would have been expected in the case of indirect RSO effect via shifts in the metabolic pathways, leading to substrate limitations for RCC.

\section{Discussion}

This study provides compelling evidence that the RCC clade of Thermoplasmata is a novel group of methylotrophic methanogens in the bovine rumen with MAs as their major energy and carbon sources. It is currently unclear whether RCC are also hydrogenotrophic or not, however, our data show that RCC occupy a niche separate from the other rumen methanogens. Nevertheless, this study showed that RCC, rather than solely hydrogenotrophic methanogens, are the primary target of $\mathrm{CH}_{4}$ mitigation via RSO supplementation. Because the RCC clade has been detected in many ruminants worldwide ${ }^{3}$, our findings suggest new possibilities for $\mathrm{CH}_{4}$ mitigation strategies for dairy cows, either by targeting the RCC directly or via dietary supplements focusing on $\mathrm{MA}$, and not on $\mathrm{H}_{2}$, for mitigating ruminal $\mathrm{CH}_{4}$ production. Still, long-term feeding experiments are needed to uncover the true potential of RCC as targets for $\mathrm{CH}_{4}$ mitigation strategies.

The identification of methanogenic organisms in the Thermoplasmata branch of the archaeal phylum Euryarchaeota in this study and other studies ${ }^{10,19}$ published during the review process of this work substantiates previous theories about the widespread occurrence of this potentially primordial metabolism that probably developed early in evolution and was in the common ancestor of all Euryarchaeota ${ }^{24}$, perhaps even of all archaea. It is also noteworthy that this branch of Thermoplasmata harbours several other groups devoid of cultivated representatives, which have been detected in rice field soils, marine sediments and other presumably anoxic habitats ${ }^{7}$, indicating a previously unrecognized, wider ecological distribution of methanogenic archaea ${ }^{19}$.

\section{Methods}

Diets and experimental setup. Four Holstein dairy cows were fed two different diets, a control (total fat content of $3.5 \%$ of dry matter (DM)) and a RSOsupplemented diet (supplemented with $33 \mathrm{~g}$ RSO per $\mathrm{kg}$ feed, resulting in a total fat content of $6.5 \%$ of DM; Supplementary Table S5), in a conventional $4 \times 2$ crossover design where two cows were fed the low fat-control diet and two cows were fed the fat-supplemented diet during the first period, and vice versa during second period. Each feeding period lasted for 3 weeks and the cows were fed twice daily during the entire period. The animal experiment was designed and approved by The Experimental Animal Inspectorate under The Danish Ministry of Justice (2008/561-1500), and completed with the highest consideration for the well being of the cows. However, one cow was excluded from the full experiment because of health considerations, which were not related to the feed manipulation.

Quantification of $\mathbf{C H}_{\mathbf{4}}$ emission from dairy cows. $\mathrm{CH}_{4}$ emission from the cows was quantified using custom-built transparent polycarbonate chambers

$(1.83 \times 3.82 \times 2.45 \mathrm{~m})$ located in the barn. The cows were housed individually, and quantification of $\mathrm{CH}_{4}$ and $\mathrm{CO}_{2}$ emission from each cow was performed over two times $48 \mathrm{~h}$ during the last week of each feeding period. The air flow through the chambers was measured with a mass flow meter (Teledyne Hastings Instruments, Hampton, VA, USA), and $\mathrm{CO}_{2}$ and $\mathrm{CH}_{4}$ was quantified using infrared sensors (Columbus Instruments, Columbus, OH, USA) exactly as described in Hellwing et al. ${ }^{25}$

Nucleic acid extraction and double-stranded (ds) cDNA synthesis. Rumen fluid-sampling strategy was developed from previous publications ${ }^{26-28}$, taking into account that a significant fraction of the rumen microbiota is adhered to fibers. Hence, representative samples of rumen fluid were collected at the end of each feeding period from the top and bottom part of the frontal, mid and distal section of the rumen $(25 \mathrm{ml}$ from each area) through a rumen fistula. The rumen fluid was pooled in sterile plastic bags with filter inserts (pore size $0.5 \mathrm{~mm}$, Grade Blender Bags; VWR - Bie \& Berntsen, Denmark), physically disrupted to release microorganisms from fibers into the rumen fluid, enabling sampling of rumen fluid representing both the fiber-adhered and the always occurring rumen fluid community. Finally, rumen fluid was sieved and decanted into $1.5 \mathrm{ml}$ Eppendorf tubes, snap frozen in liquid nitrogen and stored at $-80^{\circ} \mathrm{C}$ until nucleic acid (NA) extraction. NAs were extracted using a standard phenol-chloroform bead-beating procedure. RNA was further purified using the NucleoSpin RNA Clean-up XS (Macherey-Nagel). RiboLock RNase inhibitor was added to purified RNA samples. The contaminated DNA was removed from the combined RNA/DNA extracts through the use of the DNase I kit (Fermentas). The absence of DNA was verified by PCR targeting the 16S rRNA gene (Supplementary Table S6). DNase-treated RNA was purified using the MEGAclear kit (Invitrogen). Total RNA was quantified using a NanoDrop-1000 (Thermo Fisher Scientific, Waltham, MA, USA) and quality of the purified RNA was tested using the Experion RNA StdSens Analysis Kit (BioRad, Hercules, CA, USA).

The synthesis of double-stranded cDNA was performed using the SuperScript Double-Stranded cDNA Synthesis Kit (Invitrogen) as previously described ${ }^{29}$.

Sequencing and bioinformatic analysis. Sequencing of the samples was performed on the Illumina HiSeq2000 system (BGI, Shenzhen, Hong Kong) using fragmented ds cDNA ( $\approx 160 \mathrm{bp}$; paired-end sequenced from $2 \times 100 \mathrm{bp}$ read 
lengths). Paired-end reads were assembled using the SHE-RA software ${ }^{15}$. Concatenated sequences were filtered using a $60 \%$ quality confidence cutoff. Small subunit (SSU) rRNAs were analysed according to Urich et al. ${ }^{14}$; putative mRNArelated reads were analysed using Meta-Genome Rapid Annotation using Subsystems Technology, v. 3.1.2. (http://metagenomics.anl.gov/) with subsystembased annotation based on the SEED database ${ }^{30}$ and with MEGAN $4^{31}$ analysis of BlastX searches against the Genbank Refseq protein database (with $e$-value cutoff $\left.1 e^{-5}\right) . M c r$ transcripts were identified with BlastN searches against the data sets (with $e$-value cutoff $1 e^{-5}$ ) using representative gene sequences. Assembly of transcripts was done with CAP3

(ref. 32). Elongation factor $1 \alpha$ and $m c r B$ transcripts were assembled with stringent parameters -p90-o50; $m c r A$ and $m c r G$ were assembled with -p95-o50. Taxonomically, pre-binned RCC $16 \mathrm{~S}$ ribo-tags were assembled with default parameters.

McrA, EF-1 $\alpha$ and 16S rRNA phylogeny reconstruction. Sequences were downloaded from NCBI and the Functional Gene Pipeline/Repository homepage (http:// fungene.cme.msu.edu/). McrA and EF-1 $1 \alpha$ protein sequences, as well as $16 \mathrm{~S}$ rRNA genes, were aligned using clustalW2 (ref. 33) from European Bioinformatics Institute (EBI). Alignments were manually corrected in BioEdit ${ }^{34}$, and ambiguously aligned positions were removed (a $50 \%$ indel filter was applied for the EF- $1 \alpha$ alignment). Phylogenetic analyses were performed with the Maximum Likelihood (PhyML) software ${ }^{35}$ using default parameters and 100 bootstraps, and phylogenies were visualized with NJPlot ${ }^{36}$

Quantification of $\boldsymbol{m c r} \boldsymbol{A}$ gene copies by qPCR. Quantification of methanogen $m c r A$ gene copies in ds cDNA was done using the Roche Lightcycler 480 instrument (Roche, Mannheim, Germany). Each reaction $(20 \mu \mathrm{l})$ consisted of $10 \mu \mathrm{l}$ of Sybr Green I Master Mix (Roche), $1 \mu \mathrm{l}$ each of mlas for forward primer and mcrArev reverse primer $\left(10 \mathrm{pmol}^{-1} \mathrm{l}^{-1}\right.$ ) (Supplementary Table S6), $7 \mu \mathrm{l}$ of $\mathrm{ddH}_{2} \mathrm{O}$ and $1 \mu \mathrm{l}$ of ds cDNA template at a concentration of approximately $10 \mathrm{ng}^{-1} \mathrm{l}^{-1}$ (ds cDNA concentration quantified using NanoDrop-1000, Thermo Fisher Scientific). Running conditions were $95^{\circ} \mathrm{C}$ for $3.5 \mathrm{~min}$ followed by 40 cycles of $92^{\circ} \mathrm{C}$ for $30 \mathrm{~s}$, $55^{\circ} \mathrm{C}$ for $45 \mathrm{~s}$ and $72^{\circ} \mathrm{C}$ for $30 \mathrm{~s}$. Acquisition of fluorescent signal was done at $83^{\circ} \mathrm{C}$ $(15 \mathrm{~s})$ after each cycle. Product specificity and size was confirmed by melting point analysis and by agarose gel electrophoresis, respectively. Detection range for $\mathrm{mcrA}$ gene copies was between $10^{1}$ and $10^{9}$ gene copies per $\mu \mathrm{l}$ in the standards prepared for qPCR (slope: -3.362 , efficiency: 1.983 , error: 0.0124 ).

Rumen fluid incubations with TMA. Rumen fluid was sampled from a rumen fistulated Holstein breed dairy cow fed a maintenance diet ${ }^{37}$. Rumen fluid was sampled before morning feeding from the top and bottom part of the frontal, mid and distal section of the rumen (300-400 $\mathrm{ml}$ rumen fluid from each site), mixed and sieved through a sterile filter bag with a pore size of $0.5 \mathrm{~mm}$ (VWR) into a sterile 2-1 blue-cap bottle flushed with an anoxic nitrogen gas.

Rumen fluid ( $50 \mathrm{ml}$ ) was poured into each of eight sterile $\mathrm{N}_{2}$ gas flushed infusion bottles with $10 \mathrm{ml} 0.1 \mathrm{M}$ sodium phosphate buffer (anaerobic, pH 6.5). All bottles were sealed using gas-impermeable rubber stoppers and acclimated in a thermocontrolled shake incubator $\left(38^{\circ} \mathrm{C}, 30 \mathrm{~min}, 80\right.$ r.p.m.). Right at the beginning of the experiment, the bottles were flushed with a gas mix consisting of $10 \% \mathrm{H}_{2}, 10 \% \mathrm{CO}_{2}$ and $80 \% \mathrm{~N}_{2}$; TMA (Sigma-Aldrich, St. Louis, MO, USA) was added to four bottles ( $15 \mathrm{mM}$, final concentration). All bottles were again incubated $\left(38^{\circ} \mathrm{C}, 80\right.$ r.p.m.). Gas samples were taken at $0,2,4,6,8$ and $24 \mathrm{~h}$ and immediately analysed by gas chromatography using a ML GC82-22 (Mikrolab, Aarhus, Denmark) with helium as carrier gas. Data were analysed using the appurtenant PEAK359 software. $\mathrm{N}_{2}$ gas in the bottles was used as internal standard for calculating $\mathrm{H}_{2}, \mathrm{CO}_{2}$ and $\mathrm{CH}_{4}$ production in the rumen fluid. Rumen fluid was sampled simultaneously with gas samples, snap frozen in liquid nitrogen and stored at $-80^{\circ} \mathrm{C}$. The $\mathrm{pH}$ was measured in all bottles after $24 \mathrm{~h}$.

An additional 6-h in vitro incubation experiment was performed with six bottles, with rumen fluid and buffer as described above, under a $100 \% \mathrm{~N}_{2}$ atmosphere. Two bottles were supplemented with TMA $(10 \mathrm{mM})$, two bottles with TMA and bromoethanesulfonic acid $(20 \mathrm{mM})$, and two bottles represented control treatments. Gas samples were taken after $0,1,2,3,4$ and $6 \mathrm{~h}$ and analysed as described above.

Quantification of TMA in rumen samples using direct infusion Orbitrap mass spectrometer. A volume of $1 \mathrm{ml}$ rumen fluid from each cow at each diet was centrifuged in Eppendorf tubes for $15 \mathrm{~min}$ at 20000 r.p.m. at $4^{\circ} \mathrm{C}$. Approximately $800 \mu$ supernatant was transferred to new Eppendorf tubes and frozen at $-20^{\circ} \mathrm{C}$. In all, $50 \mu \mathrm{l}$ of sample was diluted with an equal amount of buffer solution ( $\mathrm{MeOH}: \mathrm{H}_{2} \mathrm{O}=50: 50,0.1 \%$ formic acid) containing $0.1 \mathrm{mg} \mathrm{ml}^{-1} 15 \mathrm{~N}$-labeled internal standard $\left(\mathrm{TMA}^{*} \mathrm{HCl}\right.$, Sigma-Aldrich). Samples were analysed with a LTQOrbitrap XL mass spectrometer equipped with a nano electrospray ion source (Thermo Fisher Scientific, Bremen, Germany) using direct infusion at a flow rate of $1 \mu \mathrm{min}^{-1}$. Spectra were recorded with the high-resolution Fourier-Transformation mass analyzer. The system was rinsed between sample injections with buffer solution for at least $20 \mathrm{~min}$.
Nucleic acid isolation from rumen fluid from in vitro incubations. Nucleic acids were isolated from $150 \mu \mathrm{l}$ rumen fluid using a standard phenol-chloroform method and bead beating. DNA was obtained from nucleic acids using the Allprep DNA/ RNA kit (Qiagen, Hildem, Germany) and quantified using Nanodrop (Thermo Fisher Scientific)

qPCR of gene copies in rumen fluid. Gene copies of total bacteria, RCC and nonRCC methanogens $16 \mathrm{~S}$ rRNA genes, $m c r A$ genes of all methanogens and the MA methyltransferase gene $(m t t B)$ genes of the RCC were determined by qPCR using primers summarized in Supplementary Table S6. Primers for $m t t B$ were designed based on metatranscriptome reads. Cloning and sequencing of PCR amplicons confirmed their specificity. Primers $958 \mathrm{~F}$ and $1100 \mathrm{AR}$ were used to quantify $16 \mathrm{~S}$ rRNA of non-RCC methanogens (Methanobrevibacter, Methanomicrobium, Methanobacterium, Methanomicrococcus and Methanosphera). These primers do not amplify RCC because of 1 and $3 \mathrm{bp}$ mismatches ${ }^{28}$. qPCR was performed using a Mastercycler ep realplex (Eppendorf, Wien, Austria). Reaction mixtures ( $20 \mu \mathrm{l}$ ) contained $10 \mu \mathrm{l}$ Qiagen QuantiFast SYBR Green PCR mix, $1 \mu \mathrm{l}$ of each of the specific primers at a final concentration of $0.25 \mu \mathrm{M}$ and $1 \mu \mathrm{l}$ of template DNA. Running conditions were $95^{\circ} \mathrm{C}$ for $5 \mathrm{~min}$ followed by 40 cycles of $95^{\circ} \mathrm{C}$ for $10 \mathrm{~s}$, $60^{\circ} \mathrm{C}$ for $15 \mathrm{~s}$ and $72{ }^{\circ} \mathrm{C}$ for $30 \mathrm{~s}$. Gene copies were calculated per $\mu$ g DNA. For generation of plasmids to determine standard curves, the RCC $16 \mathrm{~S}$ rRNA gene, $m c r A$ and $m t t B$, were amplified from rumen fluids, and PCR products cloned using pGemT-Easy Cloning (Promega, Fitchburg, WI, USA). For generation of standard curves of bacterial 16S rRNA and non-RCC methanogens, long fragments of PCR amplified 16S rRNA genes were used. Plasmids were linearized; plasmids and PCR products were diluted serially in 10-fold and applied as template in qPCR reactions. Melting curve analysis and agarose gel electrophoresis were performed to verify the identity of the genes of interest.

Statistical analysis. Data were statistically analysed using SigmaPlot (SigmaPlot 11; Systat Software) or Graph Pad. Effects of fat supplementation on SSU and $m c r A$ gene transcripts of RCC and Methanobacteriales, as well as on bacterial and eukaryote SSU reads, were tested using paired $t$-tests. The impact of fat supplementation on $\mathrm{CH}_{4}$ from $\mathrm{CO}_{2} / \mathrm{H}_{2}, \mathrm{CH}_{4}$ from $\mathrm{CH}_{3}-\mathrm{R}$ and $\mathrm{NH}_{4}^{+}$assimilation transcripts was evaluated using one-tailed paired $t$-tests. $\mathrm{CH}_{4}$ in vitro production from TMA was tested using non-paired $t$-test. Gene abundances of methanogens and bacteria during in vitro fermentations were compared with one-way ANOVA applying the Holm-Sidak all-pairwise multiple comparison. Generally, $P$-values $\leq 0.05$ and $<0.001$ were considered representing significant and highly significant effects of treatments, respectively, and $P$-values between $>0.05$ and $\leq 0.10$ to represent trend-wise effects of treatments.

\section{References}

1. Beauchemin, K. A., Kreuzer, M., O’Mara, F. \& McAllister, T. A. Nutritional management for enteric methane abatement: A review. Aus. J. Exp. Agri. 48, 21-27 (2008).

2. Steinfeld, H. et al. Livestock's Long Shadow: Environmental Issues and Options (Food and Agriculture Organization of the United Nations (FAO), 2006).

3. Janssen, P. H. \& Kirs, M. Structure of the archaeal community of the rumen. Appl. Environ. Microbiol. 74, 3619-3625 (2008).

4. Alexandratos, N. et al. World agriculture: towards 2030/2050. Interim report, Prospects for food, nutrition, agriculture and major commodity groups (Food and Agriculture Organization of the United Nations (FAO), 2006).

5. Eckard, R. J., Grainger, C. \& de Klein, C. A. M. Options for the abatement of methane and nitrous oxide from ruminant production: A review. Livestock Sci. 130, 47-56 (2010).

6. Tajima, K., Nagamine, T., Matsui, H., Nakamura, M. \& Aminov, R. I. Phylogenetic analysis of archaeal $16 \mathrm{~S}$ rRNA libraries from the rumen suggests the existence of a novel group of archaea not associated with known methanogens. FEMS Microbiol. Lett. 200, 67-72 (2001).

7. Kemnitz, D., Kolb, S. \& Conrad, R. Phenotypic characterization of Rice Cluster III archaea without prior isolation by applying quantitative polymerase chain reaction to an enrichment culture. Environ. Microbiol. 7, 553-565 (2005).

8. Attwood, G. T. et al. Exploring rumen methanogen genomes to identify targets for methane mitigation strategies. Anim. Feed Sci. Technol. 166-167, 65-75 (2011).

9. Evans, P. N. et al. Community composition and density of methanogens in the foregut of the Tammar Wallaby (Macropus eugenii). Appl. Environ. Microbiol. 75, 2598-2602 (2009).

10. Dridi, B., Fardeau, M. L., Ollivier, B., Raoult, D. \& Drancourt, M. Methanomassiliicoccus luminyensis gen. nov., sp. nov., a methanogenic archaeon isolated from human faeces. Int. J. Syst. Evol. Microbiol. 62, 1902-1907 (2012).

11. Grainger, C. \& Beauchemin, K. A. Can enteric methane emissions from ruminants be lowered without lowering their production? Anim. Feed Sci. Technol 166, 308-320 (2011).

12. Patra, A. K. Enteric methane mitigation technologies for ruminant livestock: a synthesis of current research and future directions. Environ Monit Assess 184, 1929-1952 (2012). 
13. Brask, M. et al. Methane production and digestion of different physical forms of rapeseed as fat supplement in dairy cows. J. Dairy Sci. (In press).

14. Urich, T. et al. Simultaneous assessment of soil microbial community structure and function through analysis of the meta-transcriptome. PLoS ONE 3, e2527 (2008).

15. Rodrigue, S. et al. Unlocking short read sequencing for metagenomics. PLoS ONE 5, 1-9 (2010).

16. Mitra, S. et al. Functional analysis of metagenomes and metatranscriptomes using SEED and KEGG. BMC Bioinformatics 12, S21 (2011).

17. Rother, M. \& Krzycki, J. A. Selenocysteine, pyrrolysine, and the unique energy metabolism of methanogenic archaea. Archaea 2010, 453642 (2010).

18. Gorlas, A., Robert, C., Gimenez, G., Drancourt, M. \& Raoult, D. Complete genome sequence of Methanomassiliicoccus luminyensis, the largest genome of a human-associated Archaea species. J. Bacteriol. 194, 4745 (2012).

19. Paul, K., Nonoh, J. O., Mikulski, L. \& Brune, A. 'Methanoplasmatales,' thermoplasmatales-related archaea in termite guts and other environments, are the seventh order of methanogens. Appl. Environ. Microbiol. 78, 8245-8253 (2012).

20. Neill, A. R., Grime, D. W. \& Dawson, R. M. C. Conversion of choline methyl groups through trimethylamine into methane in the rumen. Biochem. J. 170, 529-535 (1978).

21. Mitchell, A. D., Chappell, A. \& Knox, K. L. Metabolism of betaine in the ruminant. J. Anim. Sci. 49, 764-774 (1979).

22. Möller, B., Hippe, H. \& Gottschalk, G. Degradation of various amine compounds by mesophilic clostridia. Arch. Microbiol. 145, 85-90 (1986).

23. Bradbeer, C. The clostridial fermentations of choline and ethanolamine. J. Biol. Chem. 240, 4669-4674 (1965).

24. Bapteste, É., Brochier, C. \& Boucher, Y. Higher-level classification of the archaea: evolution of methanogenesis and methanogens. Archaea 1, 353-363 (2005).

25. Hellwing, A. L. F., Lund, P., Weisbjerg, M. R., Brask, M. \& Hvelplund, T. Technical note: Test of a low cost and animal-friendly system for measuring methane emissions from dairy cows. J. Dairy Sci. 95, 6077-6085 (2012).

26. Christophersen, C. T., Wright, A. D. G. \& Vercoe, P. E. In vitro methane emission and acetate:propionate ratio are decreased when artificial stimulation of the rumen wall is combined with increasing grain diets in sheep. J. Anim. Sci. 86, 384-389 (2008).

27. Denman, S. E., Tomkins, N. W. \& McSweeney, C. S. Quantitation and diversity analysis of ruminal methanogenic populations in response to the antimethanogenic compound bromochloromethane. FEMS Microbiol. Ecol. 62, 313-322 (2007).

28. Tymensen, L. D. \& McAllister, T. A. Community structure analysis of methanogens associated with rumen protozoa reveals bias in universal archaeal primers. Appl. Environ. Microbiol. 78, 4051-4056 (2012).

29. Radax, R. et al. Metatranscriptomics of the marine sponge Geodia barretti: tackling phylogeny and function of its microbial community. Environ. Microbiol. 14, 1308-1324 (2012).

30. Overbeek, R. et al. The subsystems approach to genome annotation and its use in the project to annotate 1000 genomes. Nucleic Acids Res. 33, 5691-5702 (2005).

31. Huson, D. H., Mitra, S., Ruscheweyh, H. J., Weber, N. \& Schuster, S. C. Integrative analysis of environmental sequences using MEGAN4. Genome Res. 21, 1552-1560 (2011).
32. Huang, X. \& Madan, A. CAP3: A DNA sequence assembly program. Genome Res. 9, 868-877 (1999).

33. Larkin, M. A. et al. ClustalW and ClustalX version 2. Bioinformatics 23, 2947-2948 (2007).

34. Hall, T. A. BioEdit: a user-friendly biological sequence alignment editor and analysis program for Windows 95/98/NT. Nucleic Acids Symp. Ser. 41, 95-98 (1999).

35. Guindon, S. \& Gascuel, O. A simple, fast, and accurate algorithm to estimate large phylogenies by maximum likelihood. Syst. Biol. 52, 696-704 (2003).

36. Perrière, G. \& Gouy, M. WWW-Query: An on-line retrieval system for biological sequence banks. Biochemie 78, 364-369 (1996).

37. Madsen, J. et al. The AAT/PBV protein evaluation system for ruminants-a revision. Norwegian J. Agri. Sci. 19, 1-37 (1995).

\section{Acknowledgements}

We thank the skillful assistance of M. Brask, A.L.F. Hellwing and T.N. Jakobsen (Tjele) during the in vivo feeding experiment. We also thank T. Rattei and T. Weinmaier (Vienna) for their help in sequence analysis. This study was financially supported by the Ministry for Food, Agriculture and Fisheries, the Faculty of Science and Technology, Aarhus University, Denmark and by the Austrian Federal Ministry of Science and Research (GEN-AU III InflammoBiota).

\section{Author contributions}

The study was designed by M.P. and T.U. Samples were collected by M.P. DNA/RNA extraction and qPCR was performed by M.P. and C.S. The ds cDNA synthesis was performed by M.P. and G.M. Analysis of Illumina sequencing data was performed by T.U., M.P. and C.S. mcrA, 16 S rRNA and EF-1 $\alpha$ phylogenies constructed by A.Sp., M.P. and T.U. In vitro incubations and $\mathrm{qPCR}$ analyses were conducted by M.P. and C.S. L.F. and W.W. quantified TMA concentrations. Statistical analysis was done by C.S., M.P. and T.U., assisted by N.C. The manuscript was written by M.P., C.S. and T.U., assisted by all co-authors.

\section{Additional information}

Accession codes: Illumina raw sequence data is available through NCBI (Bioproject: PRJNA176598; Sequence Read Archive: SRA059441).

Supplementary Information accompanies this paper on http://www.nature.com/ naturecommunications

Competing financial interests: The authors declare no competing financial interests.

Reprints and permission information is available online at http://npg.nature.com/ reprintsandpermissions/

How to cite this article: Poulsen $\mathrm{M}$ et al. Methylotrophic methanogenic Thermoplasmata implicated in reduced methane emissions from bovine rumen. Nat. Commun. 4:1428 doi: $10.1038 /$ ncomms2432 (2013).

(c) (1) (2) This work is licensed under a Creative Commons AttributionBY NA SA NonCommercial-ShareAlike 3.0 Unported License. To view a copy of this license, visit http://creativecommons.org/licenses/by-nc-sa/3.0/ 


\section{Erratum: Methylotrophic methanogenic}

\section{Thermoplasmata implicated in reduced methane emissions from bovine rumen}

Morten Poulsen, Clarissa Schwab, Bent Borg Jensen, Ricarda M. Engberg, Anja Spang, Nuria Canibe, Ole Højberg, Gabriel Milinovich, Lena Fragner, Christa Schleper, Wolfram Weckwerth, Peter Lund, Andreas Schramm \& Tim Urich

Nature Communications 4:1428 doi: 10.1038/ncomms2432 (2013); Published 5 Feb 2013; Updated 6 Jun 2013

During the production of this Article, the $y$ axis of the left-hand bar chart in Fig. 1 was mislabelled with \%o. In addition, the $P$ value in Fig. $3 \mathrm{~b}$, fourth set of bars, was displaced to the top of the panel. The correct versions of both Figs 1 and 3 appear below.
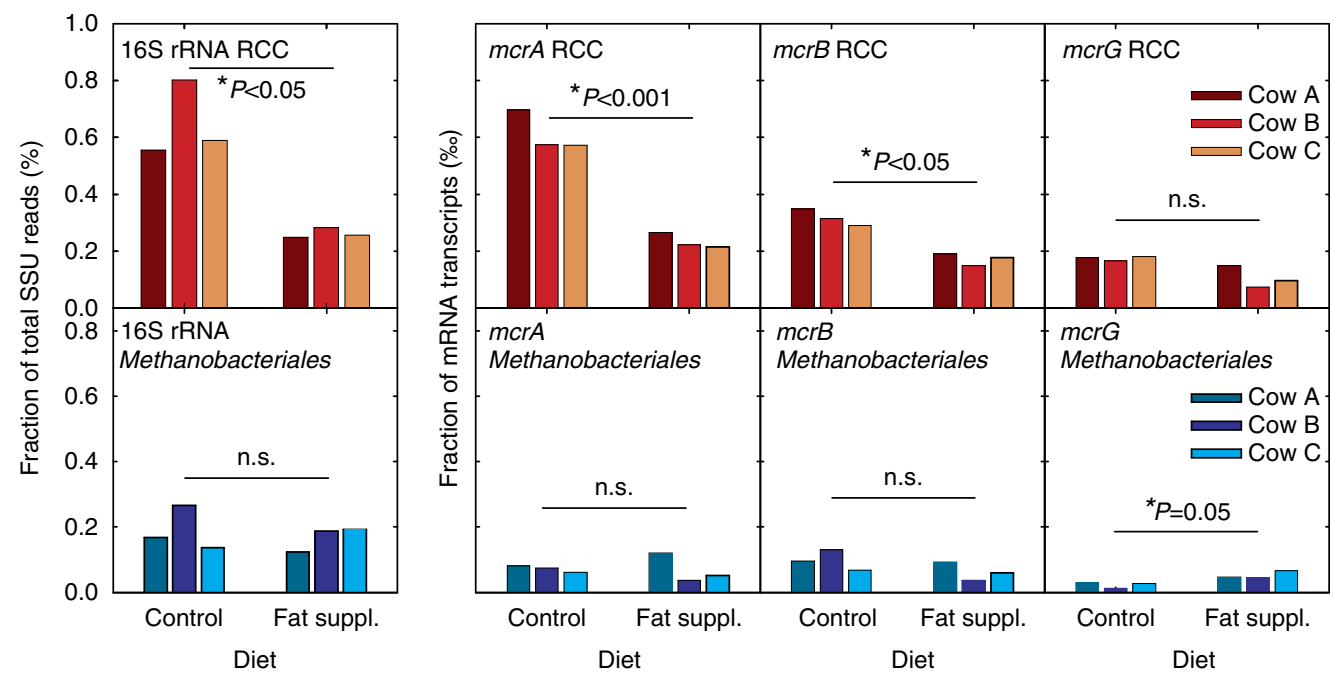

Figure 1 

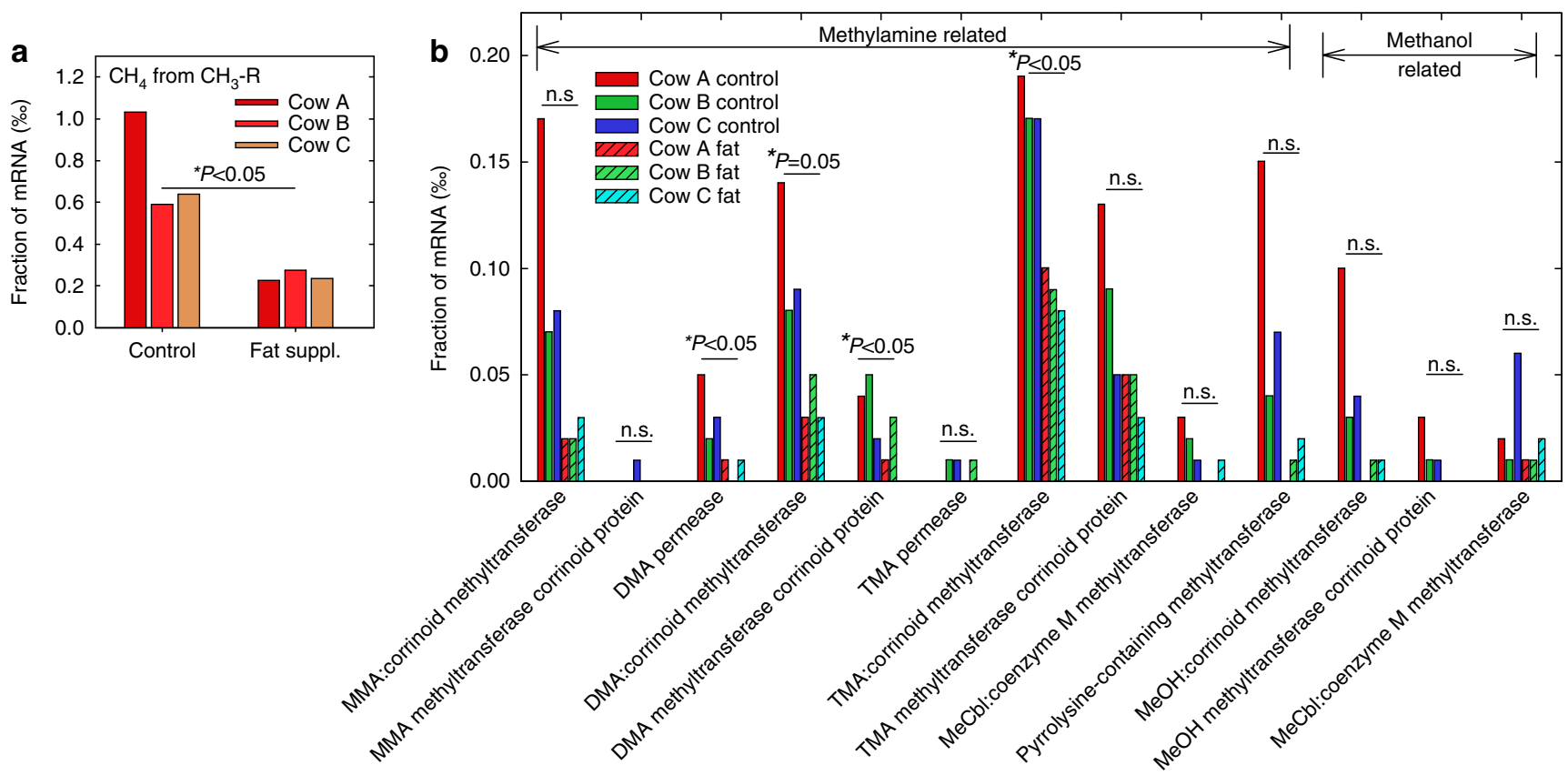

Figure 3 